\title{
On Monotone Asymptotic Pointwise Nonexpansive Mappings in Modular Function Spaces
}

\author{
B. A. Bin Dehaish (ID \\ Department of Mathematics, Faculty of Science, University of Jeddah, Jeddah 21589, Saudi Arabia \\ Correspondence should be addressed to B. A. Bin Dehaish; bbindehaish@yahoo.com
}

Received 5 November 2018; Revised 6 January 2019; Accepted 12 March 2019; Published 24 April 2019

Academic Editor: Alberto Fiorenza

Copyright (c) 2019 B. A. Bin Dehaish. This is an open access article distributed under the Creative Commons Attribution License, which permits unrestricted use, distribution, and reproduction in any medium, provided the original work is properly cited.

In this work, we investigate the existence of the fixed points of a monotone asymptotic pointwise nonexpansive mapping defined in a modular function space. Our result extends the fixed point result of Khamsi and Kozlowski.

\section{Introduction}

In the light of the three main fixed point theorems [1-3], Goebel and Kirk [4] came up with the concept of asymptotic nonexpansive mappings. Nonexpansive mappings are a particular case of asymptotic nonexpansive mappings. But the study of the existence of their fixed points appears to be extremely difficult. Kirk [5, 6] initiated the concept of pointwise Lipschitz mappings, which naturally extends the class of Lipschitz mappings. The monotone mapping fixed point theory is quite recent and attracted a lot of attention. It began with the study of Ran and Reurings [7], which extended the classical principle of Banach Contraction in partially ordered metric spaces. We suggest a recent survey for interested readers [8]. Carl and Heikkila's book [9] offers a wonderful source of monotonous mappings applications. The theory of fixed points in modular function spaces (MFS) is rooted in Khamsi, Kozlowski, and Reich's original work [10]. The Kozlowski book [11] and the recent Khamsi and Kozlowski book [12] are very important references to this subarea.

In this work, we investigate the existence of fixed points of a monotone asymptotic pointwise mappings defined in MFS. In particular, we generalize the classical fixed point result of Kirk and $\mathrm{Xu}[13]$.

\section{Preliminaries}

Extensively, details of MFS appeared in the literature; therefore, for additional information, we refer the readers to the books $[11,14]$.
Let $A$ be a nonempty set such that

(i) $\Sigma$ is a nontrivial $\sigma$-algebra of subsets of $A$;

(ii) $\mathscr{P} \subset \Sigma$ a $\delta$-ring such that $P \cap S \in \mathscr{P}$ for any $P \in \mathscr{P}$ and $S \in \Sigma$;

(iii) $A=\bigcup A_{n}$, where $\left\{A_{n}\right\} \subset \mathscr{P}$ is an increasing sequence.

Denote by $\mathscr{E}_{s}$, the vector space of simple functions whose support is in $\mathscr{P}$. Next we consider $\mathscr{M}_{\infty}$ the space of all real valued functions $f: A \longrightarrow[-\infty, \infty]$ such that there exists a sequence of simple functions $\left\{f_{n}\right\}$ which satisfy $\sup _{n \in \mathbb{N}}\left|f_{n}\right| \leq$ $|f|$, and $\lim _{n \rightarrow \infty} f_{n}(t)=f(t)$, for all $t \in A$.

Definition 1 (see $[11,14])$. A regular modular function $\varrho$ : $\mathscr{M}_{\infty} \longrightarrow[0, \infty]$ is an even function which satisfies the following conditions:

(i) $\varrho(f)=0$ implies $f=0$;

(ii) $\varrho$ is monotone; i.e., $|h(t)| \leq|H(t)|$ for all $t \in A$ implies $\varrho(h) \leq \varrho(H)$

(iii) $\left|H_{n}(t)\right| \uparrow|H(t)|$ for all $t \in A$ implies $\varrho\left(H_{n}\right) \uparrow \varrho(H)$.

We will assume throughout that function modulars are convex and regular. A subset $B \in \Sigma$ is said to be $\varrho$-null if

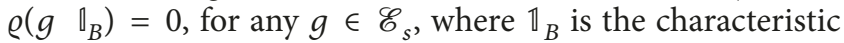
function of the subset $B$. This will allow us to say that a property holds $\varrho$-almost everywhere, and write $\varrho$-a.e., if the 
set where it does not hold is $\varrho$-null. Consider the set $\mathscr{M}=$ $\left\{f \in \mathscr{M}_{\infty} ;|f(t)|<\infty \varrho-a . e\right\}$. The MFS $L_{\varrho}$ is given by

$$
L_{\varrho}=\left\{f \in \mathscr{M} ; \lim _{\lambda \rightarrow 0} \varrho(\lambda f)=0\right\} .
$$

In the next theorem, we will review the most fundamental properties of the MFS needed in our work.

Theorem 2 (see [11, 14]). Let @ be a function modular.

(1) If $\varrho\left(\alpha h_{n}\right) \longrightarrow 0$, for some $\alpha>0$, then $h_{\psi(n)} \longrightarrow 0 \varrho-$ a.e. holds for some subsequence $\left\{h_{\psi(n)}\right\}$.

(2) We have $\varrho(g) \leq \liminf _{n \rightarrow \infty} \varrho\left(g_{n}\right)$, for any sequence $\left\{g_{n}\right\}$ such that $g_{n} \longrightarrow g Q-$ a.e.

The following definition will represent the modular versions of the classical metric concepts.

Definition 3 (see $[11,14]$ ). Let $\varrho$ be a function modular.

(1) $\left\{h_{n}\right\}$ is said to $\varrho$-converge to $h$ if $\lim _{n \rightarrow \infty} \varrho\left(h_{n}-h\right)=0$. $h$ will stand for the $Q$-limit of $\left\{h_{n}\right\}$.

(2) A sequence $\left\{h_{n}\right\}$ is called $\varrho$-Cauchy if $\lim _{n, m \rightarrow \infty} \varrho\left(h_{n}-\right.$ $\left.h_{m}\right)=0$.

(3) $C \subset L_{\varrho}$ is $\varrho$-closed if and only if the $\varrho$-limit of any $Q$-convergent sequence $\left\{h_{n}\right\} \subset C$ belongs to $C$.

(4) For a nonempty subset $C$, we define its $\varrho$-diameter as

$$
\delta_{\varrho}(C)=\sup \{\varrho(f-h) ; f, h \in C\} .
$$

$C$ is $\varrho$-bounded if and only if $\delta_{\varrho}(C)<+\infty$.

Regardless the fact that the modular may not satisfy the triangle inequality, the $\varrho$-limit is unique. But $\varrho$-convergent sequences may not be $\varrho$-Cauchy. Indeed, a simple example may be found in the variable exponent space $L^{p(\cdot)}([0,+\infty))$, where the function $p$ is defined by

$$
p(x)=n, \quad x \in[n, n+1), n \in \mathbb{N} .
$$

The function modular $\varrho$ is defined by

$$
\varrho(f)=\sum_{n=0}^{\infty} \int_{[n, n+1)}|f(x)|^{n} d x .
$$

If we take

$$
f_{n}(t)=(-1)^{n} \frac{1}{2} \mathbb{1}_{[n,+\infty)}, \quad n \in \mathbb{N},
$$

then $\varrho\left(f_{n}\right)=\sum_{i=n}^{\infty}\left(1 / 2^{i}\right)$, and

$$
\begin{aligned}
\varrho & \left(f_{n}-f_{n+1}\right) \\
& =\varrho\left((-1)^{n} \frac{1}{2} \mathbb{1}_{[n,+\infty)}-(-1)^{n+1} \frac{1}{2} \mathbb{1}_{[n+1,+\infty)}\right) \\
& =\varrho\left(\frac{1}{2} \mathbb{1}_{[n,+\infty)}+\frac{1}{2} \mathbb{1}_{[n+1,+\infty)}\right) \\
& =\varrho\left(\frac{1}{2} \mathbb{1}_{[n, n+1)}+\mathbb{1}_{[n+1,+\infty)}\right),
\end{aligned}
$$

for any $n \in \mathbb{N}$. It is easy to see that

$$
\begin{gathered}
\lim _{n \rightarrow \infty} \varrho\left(f_{n}\right)=0, \\
\varrho\left(f_{n}-f_{n+1}\right)=+\infty,
\end{gathered}
$$

$n \in \mathbb{N}$.

In other words, $\left\{f_{n}\right\}$ is $\varrho$-convergent to 0 and it is not $\varrho$ Cauchy.

Note that $\varrho$-balls $B_{\varrho}(f, r)=\left\{h \in L_{\varrho} ; \varrho(f-h) \leq r\right\}$ are $\varrho$-closed. It is interesting to notice that $\varrho$-Cauchy sequences in $L_{\varrho}$ are $\varrho$-convergent; i.e., $L_{\varrho}$ is $\varrho$-complete $[11,14]$.

The next result follows easily from Theorem 2 .

Theorem 4. Let $\varrho$ be a function modular. Let $\left\{h_{n}\right\}$ be a sequence which $Q$-converges to $h$ in $L_{\rho}$. If $\left\{h_{n}\right\}$ is monotone increasing (resp., decreasing), i.e., $h_{n} \leq h_{n+1}$ Q-a.e. (resp. $h_{n+1} \leq$ $h_{n}$ Q-a.e.), for any $n \geq 1$, then $h_{n} \leq h$ Q-a.e. (resp., $h \leq h_{n} Q^{-}$ a.e.), for any $n \geq 1$.

Next we present the definition of the modular uniform convexity which is an essential tool in metric fixed point theory.

Definition 5 (see [14]). Let $\varrho$ be a function modular. Then we will say that

(i) $\varrho$ is uniformly convex (in short (UC)) if for every $R>$ 0 and $\varepsilon>0$, we have

$$
\delta_{\varrho}(R, \varepsilon)=\inf \left\{1-\frac{1}{R} \varrho\left(\frac{f+g}{2}\right) ;(f, g) \in D\right\}>0,
$$

where $D$ is the set of all $f, g \in L_{\varrho}$ such that $\varrho(f) \leq R$, $\varrho(g) \leq R$ and $\varrho(f-g) \geq \varepsilon R$;

(ii) $\varrho$ is (UUC) if there exists $\eta(s, \varepsilon)>0$ for every $s \geq 0$, $\varepsilon>0$ such that $\delta_{\varrho}(R, \varepsilon)>\eta(s, \varepsilon)>0$, for $R>s$.

Remark 6. The modular uniform convexity in Orlicz function spaces was initiated in the work of Khamsi et al. [15]. In particular, we know that the (UC) property of the modular in Orlicz spaces is satisfied if and only if the Orlicz function is (UC) $[15,16]$. An example of an Orlicz function which is $(\mathrm{UC})$ is $\varphi(t)=e^{t^{2}}-1[17,18]$.

Modular functions which are (UUC) have a similar property to the weak-compactness in Banach spaces.

Theorem 7 (see $[14,15])$. Let $\varrho$ be a (UUC) function modular. Then $\bigcap_{n \in \mathbb{N}} C_{n} \neq \emptyset$ for any sequence $\left\{C_{n}\right\}$ of nonempty $\varrho^{-}$ bounded, $Q$-closed, and convex subsets of $L_{\varrho}$ such that $C_{n+1} C$ $C_{n}$, for any $n \in \mathbb{N}$. This intersection property is known as the property $(R)$.

This property will be of huge help throughout our work. In particular, we have the following result.

Theorem 8 (see [19]). Assume that $\varrho$ is (UUC). Let $C \subset L_{\varrho}$ be $\varrho$-bounded convex $\varrho$-closed nonempty subset. Let $\left\{g_{n}\right\} \subset C$ be a monotone increasing sequence (resp., decreasing). Then 
$\bigcap_{n \in \mathbb{N}}\left\{g \in C ; \quad g_{n} \leq g \varrho-\right.$ a.e. $\} \neq \emptyset$ (resp., $\bigcap_{n \in \mathbb{N}}\{g \in C ; \quad g \leq$ $g_{n} \varrho-$ a.e. $\} \neq \emptyset$ ).

This conclusion holds because order intervals in $L_{Q}$ are convex and $\varrho$-closed combined with the property $(R)$.

Next we give the definition of the $\varrho$-type functions which will help us prove some interesting fixed point results.

Definition 9 (see [19]). Let $\varrho$ be a function modular and $C \subset$ $L_{\varrho}$ be nonempty. A function $\varphi: C \longrightarrow[0, \infty]$ is said to be a $Q$-type if for any $f \in C$, we have

$$
\varphi(f)=\limsup _{m \longrightarrow \infty} \varrho\left(g_{m}-f\right),
$$

for some sequence $\left\{g_{m}\right\}$ in $L_{\varrho}$. Any sequence $\left\{h_{n}\right\} \subset C$ such that

$$
\lim _{n \longrightarrow \infty} \varphi\left(h_{n}\right)=\inf \{\varphi(f): f \in C\}
$$

is called a minimizing sequence of $\varphi$.

The following result played a major role in the study of fixed point problems in MFS.

Lemma 10 (see [20]). Let $\varrho$ be a function modular. Assume that $\varrho$ is (UUC). Let $C \subset L_{\varrho}$ be a $\varrho$-bounded $\varrho$-closed convex nonempty subset. Let $\varphi: C \longrightarrow[0, \infty]$ be a $\varrho$-type. Then any minimizing sequence of $\varphi$ is $Q$-convergent and its $Q$-limit is independent of the minimizing sequence.

Next we give the modular definitions of monotone Lipschitzian mappings which mimic their metric equivalents. First, recall that $f$ and $g$ are said to be comparable if $f \leq g Q^{-}$ a.e. or $g \leq f \varrho$-a.e., for any $f, g \in L_{\varrho}$.

Definition 11 (see [21]). Let $C \subset L_{\varrho}$ be nonempty. A mapping $T: C \longrightarrow C$ is said to be

(1) monotone if and only if we have

$$
f \leq g \varrho \text {-a.e. } \quad \text { implies } T(f) \leq T(g) \varrho \text {-a.e., }
$$

for any $f, g \in C$;

(2) monotone asymptotically pointwise Lipschitzian if and only if $T$ is monotone and there exists a sequence of mappings $k_{n}: C \longrightarrow[0, \infty)$ such that

$$
\varrho\left(T^{n}(f)-T^{n}(g)\right) \leq k_{n}(f) \varrho(f-g),
$$

for any $n \in \mathbb{N}$, whenever $f$ and $g$ are comparable elements in $C$. If $\lim \sup _{n \rightarrow \infty} k_{n}(f)=1$, for any $f \in C$, then $T$ is monotone asymptotically pointwise nonexpansive mapping.

A point $f \in C$ is a fixed point of $T$ if and only if $T(f)=f$.

We can always assume that $\left\{k_{n}(f)\right\}$ is a decreasing sequence for any $f \in K$.

\section{Main Results}

In this section, we will extend the result of Khamsi and Kozlowski [20] to the monotone case. The first result is the pointwise formulation of the main result of [21]. A powerful tool used to prove the existence of fixed points of asymptotic pointwise $\varrho$-nonexpansive mappings will be the existence of minimum points of $\varrho$-type functions. Since $\varrho$ may fail to satisfy the triangle inequality, $\varrho$-type functions may fail to have any good continuity properties that may guarantee the existence of a minimum point. Using the conclusion of Lemma 5.1 from the book [14], we introduce the following definition.

Definition 12. Let $\varrho$ be a regular modular. We will say that $\varrho$ is type-lsc if every $\varrho$-type function $\tau$ defined on a $\varrho$ bounded, $\varrho$-closed, and convex nonempty subset of $L_{\varrho}$ is $\varrho^{-}$ lower semicontinuous, i.e.,

$$
\tau(f) \leq \liminf _{n \longrightarrow \infty} \tau\left(f_{n}\right),
$$

for any $\left\{f_{n}\right\}$ which $\varrho$-converges to $f$.

According to Lemma 5.1 from the book [14], any uniformly continuous modular $\varrho$ is type-lsc. In [19], the authors investigated the existence of a fixed point for any monotone asymptotically nonexpansive mapping in MFS. Next we prove the pointwise version of their result.

Theorem 13. Assume that $\varrho$ is (UUC) and type-lsc. Let $C \subset$ $L_{\rho}$ be $\varrho$-bounded $\varrho$-closed convex nonempty subset. Let $T$ : $C \longrightarrow C$ be $\varrho$-continuous monotone asymptotically pointwise $\varrho$-nonexpansive. Assume there exists $f_{0} \in C$ such that $f_{0}$ and $T\left(f_{0}\right)$ are comparable. Then $T$ has a fixed point comparable to $f_{0}$.

Proof. Without any loss of generality, we assume that $f_{0} \leq$ $T\left(f_{0}\right) Q$-a.e. From the monotonicity of $T$, we deduce that the sequence $\left\{T^{n}\left(f_{0}\right)\right\}$ is monotone increasing. Let

$$
C_{\infty}=\left\{f \in C ; T^{n}\left(f_{0}\right) \leq f \varrho-a . e \text { for any } n \in \mathbb{N}\right\} .
$$

Remark 14. Implies that $C_{\infty} \neq \emptyset$. Let $\varphi: C_{\infty} \longrightarrow[0,+\infty)$ be the $\varrho$-type generated by $\left\{T^{n}\left(f_{0}\right)\right\}$, i.e.,

$$
\varphi(h)=\limsup _{n \rightarrow \infty} \varrho\left(T^{n}\left(f_{0}\right)-h\right) .
$$

Note that $\varphi(h)<\infty$, for any $h \in C_{\infty}$, since $C$ is $\varrho$-bounded. Let $\varphi_{0}=\inf \left\{\varphi(h) ; h \in C_{\infty}\right\}$, and let $\left\{f_{n}\right\} \subset C_{\infty}$ be a minimizing sequence of $\varphi$. Using Lemma 10, we conclude that $\left\{f_{n}\right\} \varrho$-converges to some $f \in C_{\infty}$. Since $\varrho$ is type-lsc, we deduce that $\varphi$ is $\varrho$-lower semicontinuous. Hence we have

$$
\varphi(f) \leq \liminf _{n \longrightarrow \infty} \varphi\left(f_{n}\right)=\varphi_{0} .
$$


Therefore, we must have $\varphi(f)=\varphi_{0}$. Next, we show that $f$ is a fixed point of $T$. Fix $h \in C_{\infty}$. Since $T$ is monotone, we have $T^{m}(h) \in C_{\infty}$ and

$$
\begin{aligned}
\varphi\left(T^{m}(h)\right) & =\limsup _{n \rightarrow \infty} \varrho\left(T^{n}\left(f_{0}\right)-T^{m}(h)\right) \\
& \leq \limsup _{n \rightarrow \infty} k_{m}(h) \varrho\left(T^{n-m}\left(f_{0}\right)-h\right) \\
& =k_{m}(h) \varphi(h),
\end{aligned}
$$

for any $m \geq 1$. In other words, the inequality $\varphi\left(T^{m}(h)\right) \leq$ $k_{m}(h) \varphi(h)$ is satisfied for any $h \in C_{\infty}$ and $m \geq 1$. Therefore, we have

$$
\varphi_{0} \leq \varphi\left(T^{m}(f)\right) \leq k_{m}(f) \varphi(f)=k_{m}(f) \varphi_{0}
$$

for any $m \geq 1$. Since $T$ is asymptotically pointwise $\varrho^{-}$ nonexpansive, we have $\lim _{m \rightarrow \infty} k_{m}(f)=1$, which implies that $\left\{T^{m}(f)\right\}$ is a minimizing sequence of $\varphi$. Using Lemma 10, we conclude that $T^{m}(f) \varrho$-converges to $f$. Since $T$ is $\varrho$ continuous $T^{m+1}(f)$ will $\varrho$-converge to $T(f)$. The uniqueness of the $\varrho$-limit implies $T(f)=f$; i.e., $f$ is a fixed point of $T$. Since $f \in C_{\infty}$, we get $f_{0} \leq f \varrho-$ a.e.

In the proof of Theorem 13, the assumption type-lsc is crucial to secure the existence of the minimum point of a type which happens to be the desired fixed point of the map. Therefore, if we relax the type-lsc, one expects the proof to get more complicated. In this case, we will follow the ideas developed by Khamsi and Kozlowski [20] which allowed them to prove the existence of a fixed point for asymptotic pointwise nonexpansive mapping defined in modular function spaces by using the existence of a minimizing sequence for a $\rho$-type function which is $\rho$-convergent.

Theorem 15. Assume that $\varrho$ is (UUC). Let $K \subset L_{\varrho}$ be $\varrho^{-}$ bounded $\varrho$-closed convex nonempty subset. Let $T: K \longrightarrow$ $K$ be $\varrho$-continuous monotone asymptotically pointwise $\varrho$ nonexpansive. Assume there exists $f_{0} \in K$ such that $f_{0}$ and $T\left(f_{0}\right)$ are comparable. Then $T$ has a fixed point comparable to $f_{0}$.

Proof. Without any loss of generality, we assume that $f_{0} \leq$ $T\left(f_{0}\right) \varrho$-a.e. As we did in the proof of Theorem 13, let $K_{\infty}=$ $\left\{f \in K ; T^{n}\left(f_{0}\right) \leq f \varrho-\right.$ a.e. and $\left.n \in \mathbb{N}\right\}$ and define the $\varrho$-type function $\varphi: K_{\infty} \longrightarrow[0,+\infty)$ generated by $\left\{T^{n}\left(f_{0}\right)\right\}$, i.e.,

$$
\varphi(h)=\limsup _{n \rightarrow \infty} \varrho\left(T^{n}\left(f_{0}\right)-h\right) .
$$

Set $\varphi_{0}=\inf \left\{\varphi(h) ; h \in K_{\infty}\right\}$. Let $\left\{f_{n}\right\} \subset K_{\infty}$ be a minimizing sequence of $\varphi$. As we did before, we know that $\left\{f_{n}\right\} \varrho$-converges to some $f \in K_{\infty}$. Since we do not know that $\varphi$ is $\varrho$-lower semicontinuous, we may not be able to show that $f$ is a minimum point of $\varphi$. Recall that we have $\varphi\left(T^{m}(h)\right) \leq k_{m}(h) \varphi(h)$, for any $h \in K_{\infty}$ and $m \geq 1$, which implies

$$
\varphi\left(T^{m}\left(f_{n}\right)\right) \leq k_{m}\left(f_{n}\right) \varphi\left(f_{n}\right)
$$

for any $n, m \geq 1$. Next, we build by induction an increasing sequence of integers $\left\{M_{n}\right\}$, such that

$$
k_{m}\left(f_{M_{n}}\right) \leq 1+\frac{1}{M_{n}}
$$

for any $n \geq 1$ and $m \geq M_{n}$. Set $M_{1}=1$. Since $\lim _{m \rightarrow \infty} k_{m}\left(f_{M_{1}}\right)=1$, there exists $M_{2}>M_{1}$ such that

$$
k_{m}\left(f_{M_{1}}\right) \leq 1+\frac{1}{M_{1}},
$$

for all $m \geq M_{2}$. Again since $\lim _{m \rightarrow \infty} k_{m}\left(f_{M_{2}}\right)=1$, there exists $M_{3}>M_{2}$ such that

$$
k_{m}\left(f_{M_{2}}\right) \leq 1+\frac{1}{M_{2}},
$$

for all $m \geq M_{3}$. By induction, we build the sequence $\left\{M_{n}\right\}$ in $\mathbb{N}$ such that $M_{n}<M_{n+1}$ and

$$
k_{m}\left(f_{M_{n}}\right) \leq 1+\frac{1}{M_{n}},
$$

for all $m \geq M_{n}$ and $n \geq 1$. For any $n \geq 1$ and $p \in \mathbb{N}$, take $m=M_{n+1}+p$. Hence

$$
\begin{aligned}
\varphi\left(T^{m}\left(f_{M_{n}}\right)\right) & \leq k_{m}\left(f_{M_{n}}\right) \varphi\left(f_{M_{n}}\right) \\
& \leq\left(1+\frac{1}{M_{n}}\right) \varphi\left(f_{M_{n}}\right) .
\end{aligned}
$$

Note that we have $\lim _{n \rightarrow \infty} M_{n}=\infty$. Therefore, if we let $n \longrightarrow$ $\infty$, we get

$$
\varphi_{0} \leq \limsup _{n \longrightarrow \infty} \varphi\left(T^{M_{n+1}+p}\left(f_{M_{n}}\right)\right) \leq \varphi_{0} .
$$

Therefore, $\left\{T^{M_{n+1}+p}\left(f_{M_{n}}\right)\right\}$ is a $\varrho$-minimizing sequence of $\varphi$. Using Lemma 10 , we conclude that $\left\{T^{M_{n+1}+p}\left(f_{M_{n}}\right)\right\}$ is $\varrho$-convergent to $f$ for any $p \in \mathbb{N}$. Take $p \stackrel{n}{=} 0$; we get the sequence $\left\{T^{M_{n+1}}\left(f_{M_{n}}\right)\right\}$ is $\varrho$-convergent to $f$. Using the $\varrho$-continuity of $T$, we get $\left\{T\left(T^{M_{n+1}}\left(f_{M_{n}}\right)\right)\right\}$ is $\varrho^{-}$ convergent to $T(f)$. Using the uniqueness of the $\varrho$-limit and $T\left(T^{M_{n+1}}\left(f_{M_{n}}\right)\right)=T^{M_{n+1}+1}\left(f_{M_{n}}\right)$, we conclude that $T(f)=f$; i.e., $f$ is a fixed point of $T$. Since $f \in K_{\infty}$, we have $f_{0} \leq$ $f \varrho-a . e$ as claimed.

Remark. Examples of asymptotically nonexpansive mappings are not easily found. As it was pointed out by Kirk and $\mathrm{Xu}$ [13], the original example given by Goebel and Kirk may be modified to generate an example of a monotone asymptotically nonexpansive mapping. Indeed, let $C$ be the positive part of the unite ball $B_{1}$ of $\ell_{2}$, i.e.

$$
C=\left\{\left(f_{n}\right) \in B_{1} ; f_{n} \geq 0 \text { for any } n \geq 1\right\} .
$$

Define the mapping $T: C \longrightarrow C$ by

$$
T\left(f_{n}\right)=\left(0, f_{1}^{2}, C_{2} f_{2}, C_{3} f_{3}, \cdots\right) .
$$

If we assume $C_{n} \in(0,1)$, for any $n \geq 2$, and $\prod_{n=2}^{\infty} C_{n}=$ $1 / 2$, then we can show that $T$ is a monotone asymptotically nonexpansive mapping which is not nonexpansive. 


\section{Data Availability}

No data were used to support this study.

\section{Conflicts of Interest}

The author declares that they have no conflicts of interest.

\section{References}

[1] F. E. Browder, "Nonexpansive nonlinear operators in a banach space," Proceedings of the National Acadamy of Sciences of the United States of America, vol. 54, no. 4, pp. 1041-1044, 1965.

[2] D. Göhde, "Zum prinzip der kontraktiven abbildung," Mathematische Nachrichten, vol. 30, no. 3-4, pp. 251-258, 1965.

[3] W. A. Kirk, "A fixed point theorem for mappings which do not increase distances," The American Mathematical Monthly, vol. 72, pp. 1004-1006, 1965.

[4] K. Goebel and W. A. Kirk, "A fixed point theorem for asymptotically nonexpansive mappings," Proceedings of the American Mathematical Society, vol. 35, no. 1, pp. 171-174, 1972.

[5] W. A. Kirk, "Fixed points of asymptotic contractions," Journal of Mathematical Analysis and Applications, vol. 277, no. 2, pp. 645-650, 2003.

[6] W. A. Kirk, "Asymptotic pointwise contractions," in Plenary Lecture, in Proceedings of the the 8th International Conference on Fixed Point Theory and Its Applications, Thailand, 2007.

[7] A. C. M. Ran and M. C. B. Reurings, "A fixed point theorem in partially ordered sets and some applications to matrix equations," Proceedings of the American Mathematical Society, vol. 132, no. 5, pp. 1435-1443, 2004.

[8] M. Bachar and M. A. Khamsi, "Recent contributions to fixed point theory of monotone mappings," Journal of Fixed Point Theory and Applications, vol. 19, no. 3, pp. 1953-1976, 2017.

[9] S. Carl and S. Heikkilä, Fixed Point Theory in Ordered Sets and Applications: From Differential and Integral Equations to Game Theory, Springer, Berlin, New York, 2011.

[10] M. A. Khamsi, W. M. Kozlowski, and S. Reich, "Fixed point theory in modular function spaces," Nonlinear Analysis: Theory, Methods \& Applications, vol. 14, no. 11, pp. 935-953, 1990.

[11] W. M. Kozlowski, "Modular function spaces," in Series of Monographs and Textbooks in Pure and Applied Mathematics, vol. 122, Marcel Dekker, Inc., New York, USA, 1988.

[12] M. A. Khamsi and W. M. Kozlowski, "Fixed point theory in modular function spaces," Fixed Point Theory in Modular Function Spaces, pp. 1-245, 2015.

[13] W. Kirk and H. Xu, "Asymptotic pointwise contractions," Nonlinear Analysis: Theory, Methods \& Applications, vol. 69, no. 12, pp. 4706-4712, 2008.

[14] M. A. Khamsi and W. A. Kirk, An Introdunction toMetric Spaces and Fixed Point Theory, Wiley, New York, NY, USA, 2001.

[15] M. A. Khamsi, W. M. Kozlowski, and S. T. Chen, "Some geometrical properties and fixed point theorems in Orlicz spaces," Journal of Mathematical Analysis and Applications, vol. 155, no. 2, pp. 393-412, 1991.

[16] C. Shutao, "Geometry of orlicz spaces," Dissertationes Mathematicae, vol. 356, 1996.

[17] M. A. Krasnosel'skii and Ya. B. Rutickii, Convex Functions and Orlicz Spaces, P. Noordhoff Ltd., Groningen, Netherlands, 1961.

[18] H. W. Milnes, "Convexity of orlicz spaces," Pacific Journal of Mathematics, vol. 7, no. 3, pp. 1451-1483, 1957.
[19] M. R. Alfuraidan, M. Bachar, and M. A. Khamsi, "Fixed points of monotone asymptotically nonexpansive mappings in modular function spaces," Journal of Nonlinear and Convex Analysis. An International Journal, vol. 18, no. 4, pp. 565-573, 2017.

[20] M. A. Khamsi and W. M. Kozlowski, "On asymptotic pointwise nonexpansive mappings in modular function spaces," Journal of Mathematical Analysis and Applications, vol. 380, no. 2, pp. 697-708, 2011.

[21] D. Bin and M. Khamsi, "Monotone asymptotic pointwise contractions," Filomat, vol. 31, no. 11, pp. 3291-3294, 2017. 


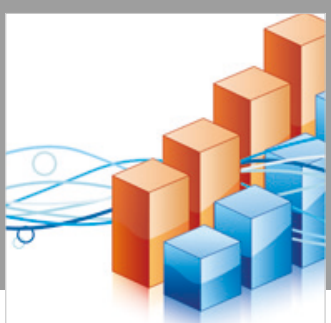

Advances in

Operations Research

\section{-n-m}
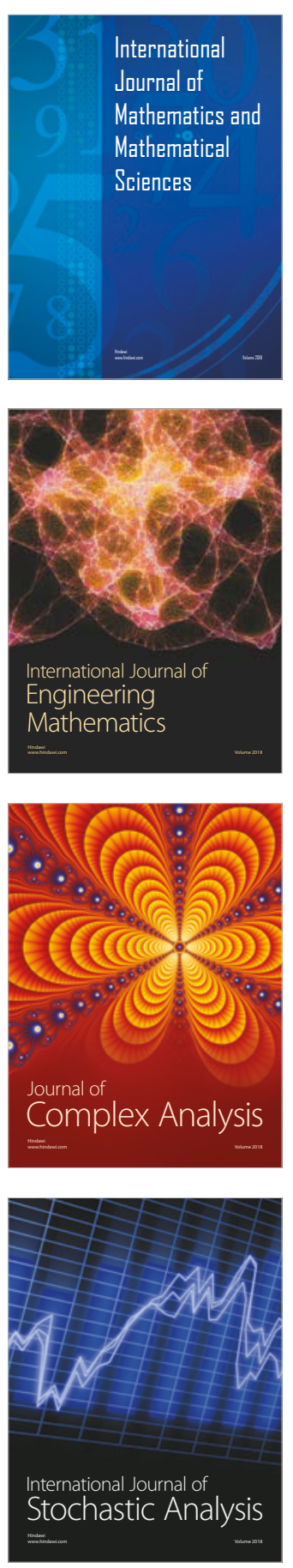
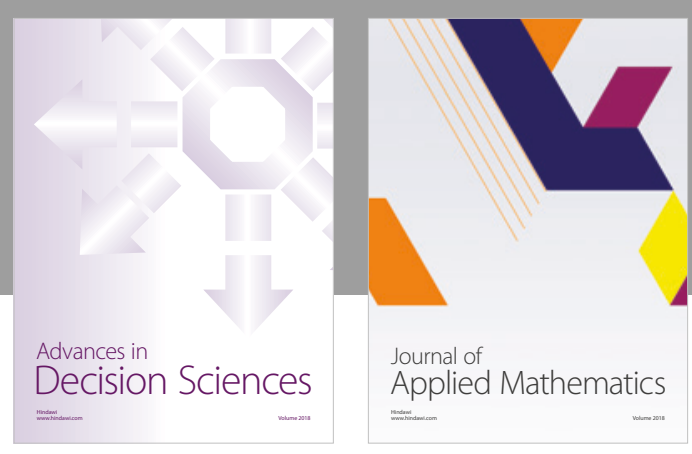

Journal of

Applied Mathematics
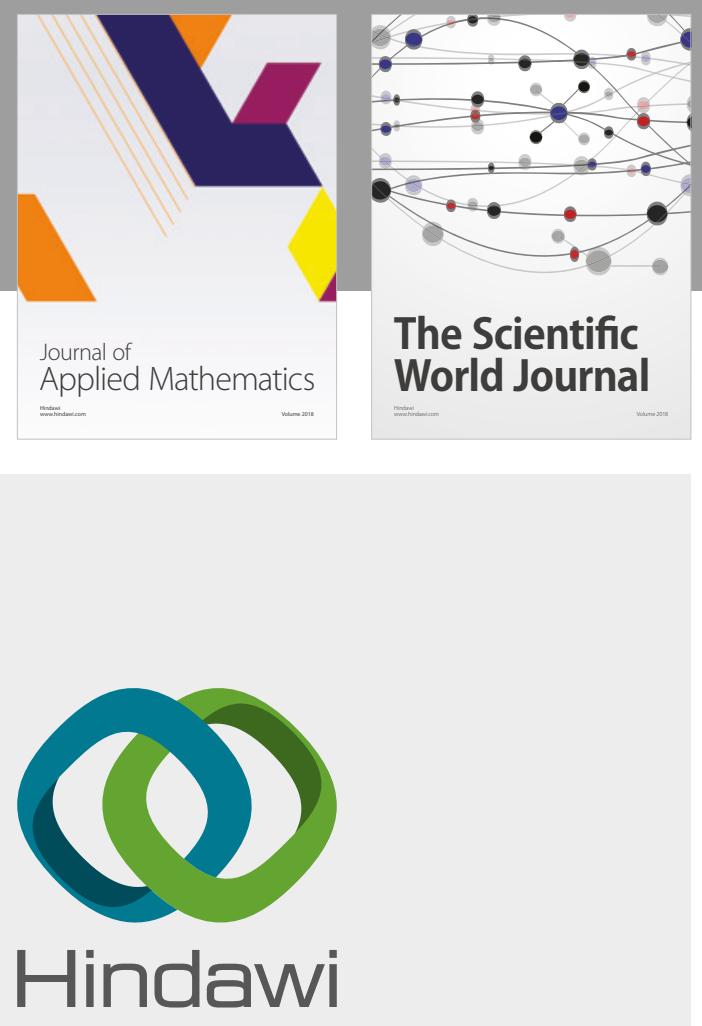

Submit your manuscripts at

www.hindawi.com

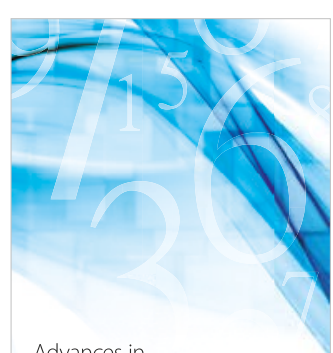

Advances in
Numerical Analysis
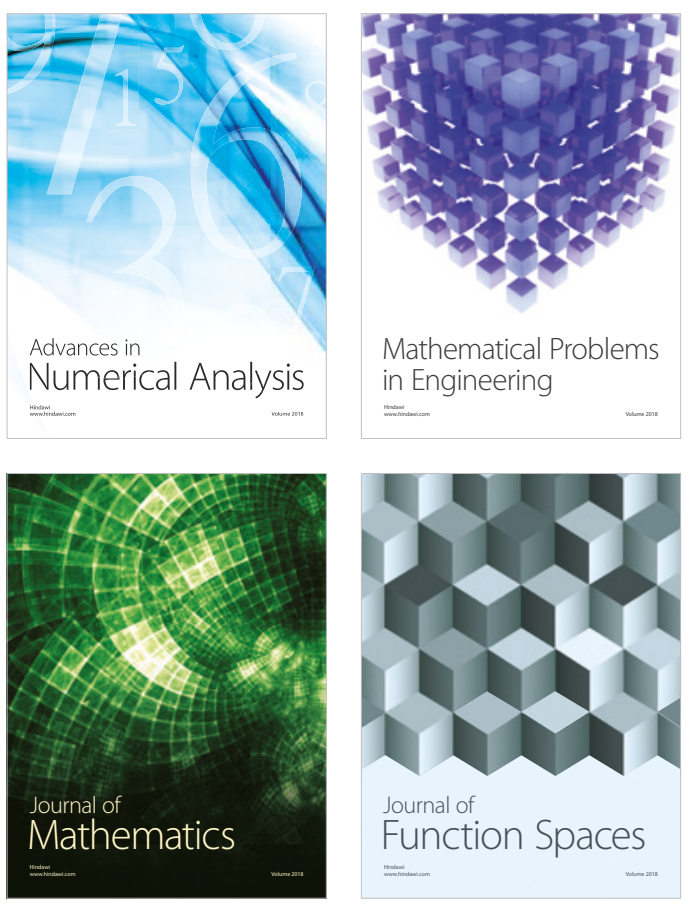

Mathematical Problems in Engineering

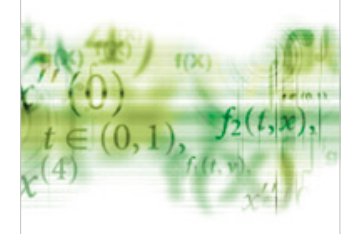

International Journal of

Differential Equations

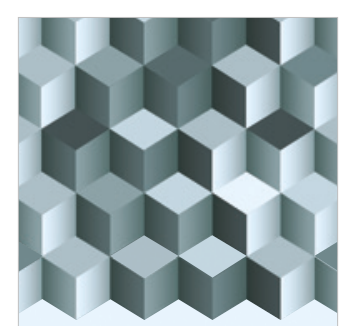

Journal of

Function Spaces

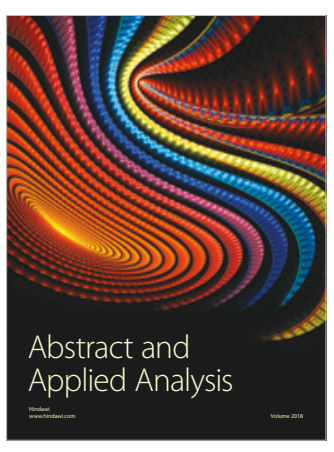

The Scientific

World Journal

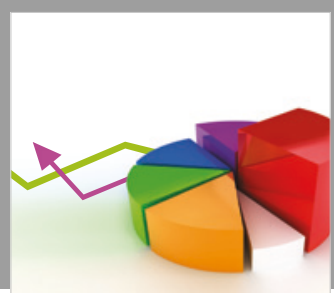

Journal of

Probability and Statistics
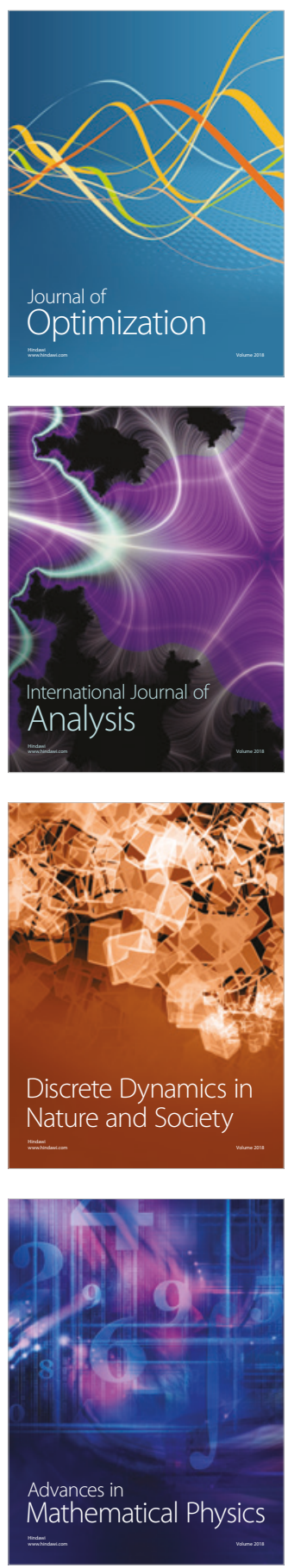